\title{
Publicidad social y su influencia en la percepción de las campañas sociales de prevención de accidentes de tránsito en Ecuador
}

\section{Social advertising and its influence on the perception of social campaigns for the prevention of traffic accidents in Ecuador}

\begin{abstract}
Mg. Amy Cárdenas-Rebelo es egresada de la Maestría en Comunicación y Marketing de la Universidad del Azuay (Ecuador) (amy.cardenas.r@gmail.com) (https://orcid.org/0000-0002-9750-4904)
\end{abstract}

Dr. Jaime Alberto Orozco-Toro es profesor e investigador de la Universidad Pontificia Bolivariana (Colombia) (jaime.orozco@upb.edu.co) (https://orcid.org/0000-0002-8152-7474)

\begin{abstract}
Resumen
El presente artículo analiza las percepciones que el público adulto de la ciudad de Cuenca en Ecuador tiene sobre las campañas sociales de prevención de accidentes de tránsito. El marco teórico lo constituyen las teorías asociadas al mercadeo social y a la publicidad social, analizando la relación que existe entre los agentes de cambio y los adoptantes objetivo. La metodología se apropia de una investigación mixta, la cual permitió la aplicación de entrevistas y 400 encuestas a ciudadanos, entre los 18 y 49 años de la ciudad de Cuenca. Los objetivos fundamentales estaban dirigidos a conocer la percepción de la ciudadanía respecto a las campañas sociales de prevención de accidentes, identificar el nivel de conciencia ciudadana respecto a la problemática de los accidentes de tránsito en Ecuador, e investigar la opinión de los ciudadanos respecto a la corresponsabilidad de la seguridad vial en la ciudad. Los resultados evidencian que hay un desconocimiento generalizado sobre las campañas de prevención vial, y que, además, los mensajes emitidos por los agentes de cambio no son claros. Se concluye que el adoptante objetivo recuerda los mensajes de manera muy dispersa, no se empodera de las campañas a pesar de comprender la información de los mensajes emitidos. Finalmente, tanto agentes de cambio como adoptantes objetivo están de acuerdo que las campañas de publicidad social son necesarias para cambiar la conducta pública, disminuir la accidentalidad y mejorar la calidad de vida de los ciudadanos.
\end{abstract}

\begin{abstract}
The present article analyzes the perceptions that the adult public of Cuenca city in Ecuador has about the social campaigns of traffic accident prevention. The theoretical framework is constituted by the theories associated with social marketing and social advertising, analyzing the relationship that exists between change agents and target adopters. The methodology consisted of a mixed research, allowing the administration of interviews and 400 surveys to citizens, between 18 and 49 years of Cuenca city. The fundamental objectives were aimed at knowing the perception of the citizenship regarding the social campaigns of accident prevention, discovering the level of citizen awareness towards the problem of traffic accidents in Ecuador, and investigating people's opinion about the co-responsibility for road safety in the city. The results showed that there is a general lack of knowledge about road prevention campaigns, and that, in addition, the messages issued by agents of change are not clear enough. In conclusion, the target adopter remembers the messages in a very dispersed way, and does not get empowered either with the campaigns despite understanding the information of the messages issued. Finally, both change agents and target adopters agree that social advertising campaigns are necessary to change public behavior, reduce accidents and improve citizens' quality of life.
\end{abstract}

\section{Palabras clave I keywords}

Publicidad social, mercadeo social, campañas sociales, accidentes de tránsito, Ecuador. Social advertising, social marketing, social campaigns, traffic accidents, Ecuador.

Cómo citar: Cárdenas-Rebelo, A., y Orozco-Toro J.A (2020). Percepción de las campañas sociales de prevención de accidentes de tránsito en Ecuador. Retos Revista de Ciencias de la Administración y Economia, 10(20), pp. 219-231. https://doi.org/10.17163/ret.n20.2020.02 


\section{Introducción}

La publicidad social (PS) ha venido adquiriendo cada vez más importancia en el ámbito de la comunicación, debido a múltiples circunstancias, entre ellas, las más relevantes están relacionadas con la gran cantidad de problemas sociales que aquejan a la sociedad, y por la necesidad de mejorar la calidad de vida de los ciudadanos.

Una de las dificultades que ha tenido el desarrollo de la PS en América Latina es que las teorías y los avances conceptuales han estado rezagados, pues se le ha dado prelación a la práctica de las campañas, por encima de una conceptualización que permita construir una teoría con fuertes bases epistemológicas. Sin embargo, en los últimos años la PS ha venido convirtiéndose en objeto de investigación por parte de importantes teóricos como Kotler y Roberto (1992), Muñoz (2001), Benet y NosAldás (2003), Barranco (2005), Eguizábal (2009), Alvarado (2012), Kotler et al. (2013), Rodrigo y Rodrigo (2013), Rosario-Ubiera (2014), entre otros.

Bajo estas perspectivas, uno de los mayores problemas de los países latinoamericanos es el alto índice de siniestralidad por accidentes de tránsito, lo que ha afectado negativamente la calidad de vida de muchas personas. En Ecuador, la mortalidad y morbilidad infantil por accidentes de tránsito alcanza cifras alarmantes, a pesar de que las medidas legales y coercitivas han tenido un efecto positivo en la reducción de mortalidad por accidentes de tránsito (Ministerio del Interior, 2014).

Por tal motivo, este artículo busca conocer algunos de los elementos teóricos fundamentales de la PS y la percepción de la sociedad respecto a las campañas de prevención de accidentes de tránsito terrestre en la ciudad de Cuenca (Ecuador).

\subsection{El mercadeo social}

La PS surge del desarrollo conceptual del mercadeo social (MS), especialmente a partir de los postulados de Kotler y Roberto (1992), quienes en su libro "Marketing social: estrategias para cambiar la conducta pública” revelan las diferencias con el mercadeo comercial. Algunos de los elementos fundamentales del MS se pueden sintetizar en los siguientes postulados: a) el enfoque está en buscar cambios de comportamiento por parte de los ciudadanos (Moliner, 1998; Pinilla, 2011); b) quienes emiten los mensajes sociales se denominan "agentes de cambio" pues buscan cambios en la conducta (Orozco-Toro \& Ferré-Pavia, 2015); c) los públicos objetivos se conocen como "adoptantes objetivo", ya que son los llamados a adoptar nuevos comportamientos y representan "individuos, grupos, poblaciones enteras, que son el objetivo de las llamadas al cambio por parte del agente del marketing social” (Kotler \& Roberto, 1992, p. 20); d) no se trabaja con productos sino con problemas a enfrentar por lo cual son las instituciones las que deben ofrecer las soluciones (Alvarado, 2005).

Además, uno de los cambios más importantes es el enfoque en la mezcla de mercadeo, pues en el MS el producto se considera un intangible pues se convierte en ideas, creencias, valores o actitudes que deben asumir los adoptantes objetivos. El precio se concibe como el esfuerzo o el tiempo que el adoptante objetivo asume para el cambio social. La promoción responde al uso de las acciones de comunicaciones integradas de mercadeo que se utilizan para difundir la campaña. Finalmente, la plaza se refiere al lugar donde se presentan los beneficios de las acciones sociales o los medios de distribución para suministrar el producto social (Kotler \& Zaltman, 1971; Gómez \& Quintanilla, 1988; French et al., 2011). 
Si bien es cierto que el MS ha sido la base fundamental para la generación de las teorías vinculadas a la PS, hoy en día se le confiere cada vez más importancia a las teorías y funciones que cumplen las campañas sociales lideradas por agentes de cambio con miras a generar nuevos comportamientos en los adoptantes objetivo.

\subsection{La publicidad social}

Se puede entender el concepto de PS como "un esfuerzo organizado, dirigido por un grupo (el agente de cambio), que intenta persuadir a otros (adoptantes objetivo) de que acepten, modifiquen, o abandonen ciertas ideas, actitudes, prácticas y conductas" (Kotler \& Roberto, 1992, p. 7). Bajo estas condiciones, se puede considerar que la PS es relativamente nueva, pues, aunque esta definición de 1992 la pone en la esfera académica, se conciben sus inicios en 1942 "cuando se ponen las tácticas de la publicidad al servicio de temas de utilidad pública alejados de cualquier interés mercantil" (Baños \& Rodríguez, 2009, p. 218). Desde otra perspectiva Gumucio-Dagron (2011) asume que el mayor desarrollo de la PS se presenta a partir de 1997 cuando la Fundación Rockefeller discutió el papel de la comunicación en los cambios sociales.

Dentro de nuestro contexto, la PS viene ganando un espacio importante en la comunicación, especialmente en los países Latinoamericanos (Pérez, 2006; Taricco, 2009; Vergara \& Rodríguez, 2010; Orozco-Toro \& Muñoz-Sánchez, 2019). Este tipo de publicidad más informativa y educativa, que busca la participación, se hace absolutamente necesaria en la solución de las problemáticas sociales que aquejan a los países en vía de desarrollo, y a pesar que en América Latina se realicen importantes campañas sociales: "el grueso de la industria ha seguido trabajando mayoritariamente para causas comerciales, siendo la publicidad social escasa y relegada a una menor difusión en los grandes medios masivos por la escasez de presupuestos" (Alvarado, 2012, p. 199).

Las campañas de PS deben contar con una serie de condiciones necesarias para que su comunicación tenga mayor eficiencia (Orozco, 2010; Dueñas et al., 2015; López-Medina, 2019). Al respecto se pueden vislumbrar algunas de las más relevantes:

Persuasión y disuasión. Las campañas de PS deben implementar tanto la disuasión (busca que los ciudadanos abandonen comportamientos nocivos como el consumo de drogas o conducir cuando se ha ingerido licor), como una comunicación persuasiva (incentivar la donación de sangre o el uso del cinturón de seguridad cuando se conduce).

Comunicación preventiva. La mejor estrategia posible de la PS es tratar de prevenir situaciones sobre una comunicación que apele a la contingencia (prevención en el uso de drogas o advertir sobre el peligro de consumir licor y conducir).

Educación e información. Se debe buscar una comunicación que informe a los adoptantes objetivo y que los eduque (realizar revisiones técnicas y mecánicas en los automóviles para prevenir accidentes).

Intangibilidad. La PS se enfrenta a la difícil tarea de vender la idea del altruismo, solidaridad o voluntariado; tareas más complejas pues implica la movilidad y el esfuerzo de los adoptantes objetivo.

Tipo de mensaje. La PS puede asumir que los mensajes no atemoricen a los adoptantes objetivo, que se fundamenten en ideas realizables, que no se sobredimensione la realidad, y especialmente mostrar los problemas, pero también las posibles soluciones.

Es claro que la PS posee unas condiciones que la hacen diferente de su homóloga comercial, incluso en la forma en que se han abordado las últimas investigaciones 
sobre su desempeño (Leal, 2000). Desde este ámbito, los mayores esfuerzos se realizan en torno a las conductas de los ciudadanos ante la acción publicitaria (Saghi \& Lotfabadi, 2016), o los cambios en los comportamientos y en las actitudes de los adoptantes objetivo (Aydin \& Koc, 2016). En el contexto iberoamericano los mayores esfuerzos se han presentado en España, con algunos aportes puntuales en América Latina. Los estudios son variados, y se enfocan desde la eficacia de la PS en televisión (Rosario-Ubiera, 2014), los mensajes publicitarios de causas sociales en momentos de crisis (Rodrigo \& Rodrigo, 2013), la PS en las universidades (Arroyo, 2014), la retórica en la PS (Cano, 2015), o la incidencia de la PS en la imagen de una marca (OrozcoToro \& Ferré-Pavia, 2015), el abuso de lo social en la publicidad (Alvarado, 2009), comunicación para el cambio social (Dagron, 2011). La mayor dificultad de las investigaciones que se realizan en torno a la PS es que sus efectos no se pueden vislumbrar, en la mayoría de las ocasiones, en el corto plazo. Ya que la PS busca principalmente cambios en la conducta, dichos cambios se producen de forma más lenta que los que busca la publicidad comercial (Martínez, 1998). Incluso, la PS no podrá contribuir a la transformación social si no ataca las verdaderas raíces de las problemáticas sociales (Arely \& Martín, 2017).

Así es como, el comportamiento relacionado con la seguridad vial logra una transformación en cualquier grupo social, y además implicará grandes esfuerzos para el agente de cambio y un esfuerzo aún mayor para los adoptantes objetivo. Desde la perspectiva de los accidentes de tránsito, se ha demostrado que las campañas de publicidad social han logrado reducir la incidencia de las personas que conducen cuando han bebido alcohol (Sar \& Anghelce, 2015).

Las campañas de publicidad social tienen una influencia positiva cuando se desarrollan basadas en una estrategia previa, con una intención responsable (Saiz et al., 2018). En este orden de ideas, el impacto de una campaña con fines sociales no solo se debe medir por el número de personas que han donado sangre, por la cantidad de dinero recaudado para una obra benéfica o por la disminución en la accidentalidad, por poner solo tres ejemplos, sino también indagando a través de encuestas y estudios de actitudes a los diversos adoptantes objetivo.

\section{Materiales y método}

El objetivo principal de esta investigación se dirigió a conocer la percepción de los adoptantes objetivo respecto a las campañas sociales de prevención de accidentes de tránsito terrestre en la ciudad de Cuenca (Ecuador).

La metodología de investigación fue mixta. En la investigación cualitativa, se realizaron entrevistas a profundidad (instrumento de 27 preguntas), con la finalidad de conocer la percepción de la ciudadanía sobre las campañas sociales realizadas en la ciudad de Cuenca, respecto a la prevención de accidentes de tránsito y el interés de las personas respecto al tema.

En la investigación cuantitativa se aplicaron 400 encuestas (instrumento de 8 preguntas) a personas entre los 18 y 49 años de edad. El método de muestreo, por conveniencia, se realizó en lugares de alto tráfico, en donde se logró encuestar a personas de diversos niveles socioeconómicos y culturales. Según la estimación del Instituto Nacional de Estadística y Censos de Ecuador, la población aproximada de Cuenca es 
de 580706 habitantes al 2015, por lo cual se aplicaron 400 encuestas, lo que representa un margen de error del 5\% y un nivel de confianza del 95\%, por lo tanto, la muestra es estadísticamente representativa.

Los objetivos específicos de la investigación estaban dirigidos a evidenciar la percepción de la ciudadanía respecto a las campañas sociales de prevención de accidentes; identificar el nivel de conciencia ciudadana respecto a la problemática de los accidentes de tránsito en Ecuador; investigar la opinión de los ciudadanos respecto a la corresponsabilidad de la seguridad vial en la ciudad de Cuenca; y finalmente, indagar qué factor es el que consideran más importante para mejorar la seguridad vial.

\section{Resultados}

\subsection{Resultados de la investigación cuantitativa}

La encuesta se realizó mediante cuestionario, cara a cara, a 400 individuos, hombres y mujeres en el rango de edad del adoptante objetivo propuesto, de esta manera se obtuvo una muestra representativa. A continuación, se describen algunos elementos del estudio empírico (Tabla 1).

Tabla 1. Especificaciones del trabajo de campo

\begin{tabular}{|c|c|c|}
\hline Procedimiento metodológico & \multicolumn{2}{|c|}{ Encuesta presencial } \\
\hline Área geográfica & \multicolumn{2}{|c|}{ Cuenca-Ecuador } \\
\hline Total de cuestionarios & \multicolumn{2}{|l|}{400} \\
\hline Sexo de la muestra & \multicolumn{2}{|l|}{$\begin{array}{l}\text { 51\% Mujeres } \\
49 \% \text { Hombres }\end{array}$} \\
\hline \multirow{4}{*}{ Distribución de edades de muestra } & $32 \%$ & 18 a 25 años \\
\hline & $29 \%$ & 26 a 33 años \\
\hline & $25 \%$ & 34 a 41 años \\
\hline & $14 \%$ & 42 a 49 años \\
\hline \multirow{4}{*}{ Distribución del nivel de educación } & $4 \%$ & Primaria \\
\hline & $47 \%$ & Secundaria \\
\hline & $35 \%$ & Universitaria \\
\hline & $14 \%$ & Posgrado \\
\hline
\end{tabular}

Fuente: Elaboración propia.

Desarrollando las preguntas contenidas en el cuestionario, al consultarles a los encuestados, ¿cómo considera el comportamiento de la mayoría de los conductores en la ciudad? En una escala de puntuación de 1 como pésimo y 5 como excelente. Los resultados determinan que al sumar las respuestas 1 y 2 (ningún o poco respeto al peatón) sobrepasa el 50\% de encuestados, mientras menos del 10\% consideran que sí existe respeto del conductor al peatón (suma de las opciones 4 y 5). El valor promedio de esta calificación es de 2.38 . 
Figura 1. Percepción del respeto que los conductores tienen hacia el peatón

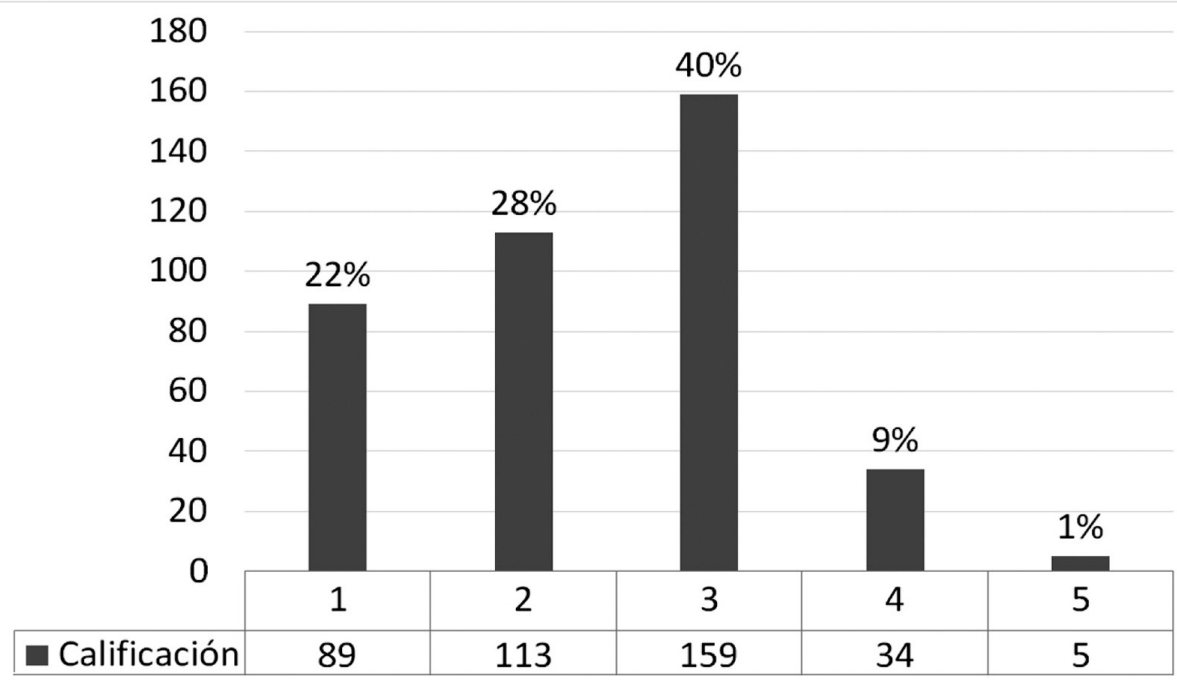

Fuente: Elaboración de los autores.

En la siguiente pregunta se indaga por la percepción que se tiene sobre el respeto que un conductor manifiesta frente a otros conductores. El 53\% de los encuestados (respuestas 1 y 2) perciben un bajo respeto entre conductores, apenas el 12\% (respuestas 4 y 5), tiene la percepción de que sí existe respeto entre conductores. El promedio obtenido de todos los encuestados es de 2.44 .

Figura 2. Percepción del respeto que los conductores tienen hacia otros conductores

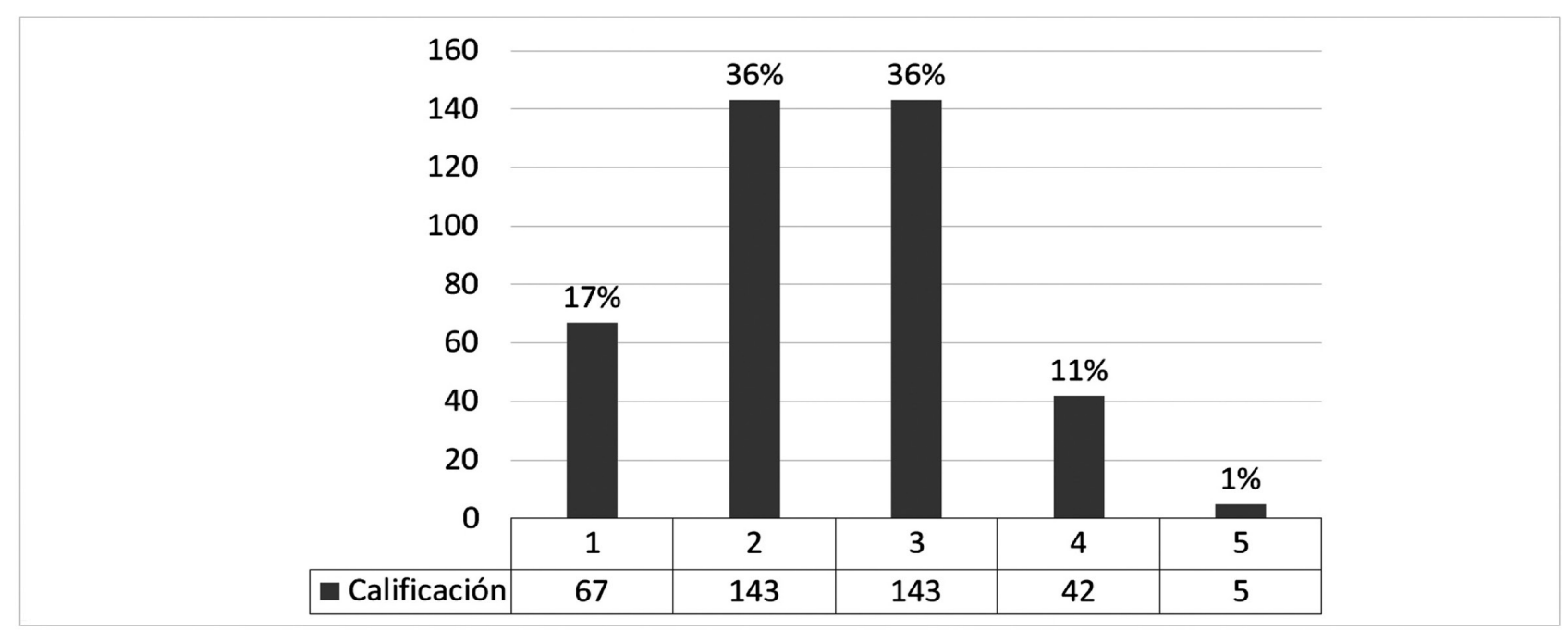

Fuente: Elaboración de los autores.

Posteriormente se pregunta por el respeto de los conductores a los pasos de cebra. Desde el punto de vista de los encuestados, se percibe que apenas el $1 \%$ de los conductores lo respetan, y por otro lado, casi el 70\% tiene un bajo o ningún respeto por esta señalización (respuestas 1 y 2). El promedio global de esta pregunta es el menor de todos los puntos evaluados con 2.06.

De igual forma, al consultar sobre la percepción del respeto que los conductores tienen a las señales de tránsito se observa que solo el 21\% (respuestas 4 y 5) de la 
población percibe que los conductores respetan adecuadamente las señales de tránsito. El $42 \%$ manifiesta que los conductores respetan muy poco o nada estas señales. El valor promedio para esta evaluación es de 2.66.

\section{Figura 3. Percepción del respeto que los conductores tienen hacia las señales de tránsito}

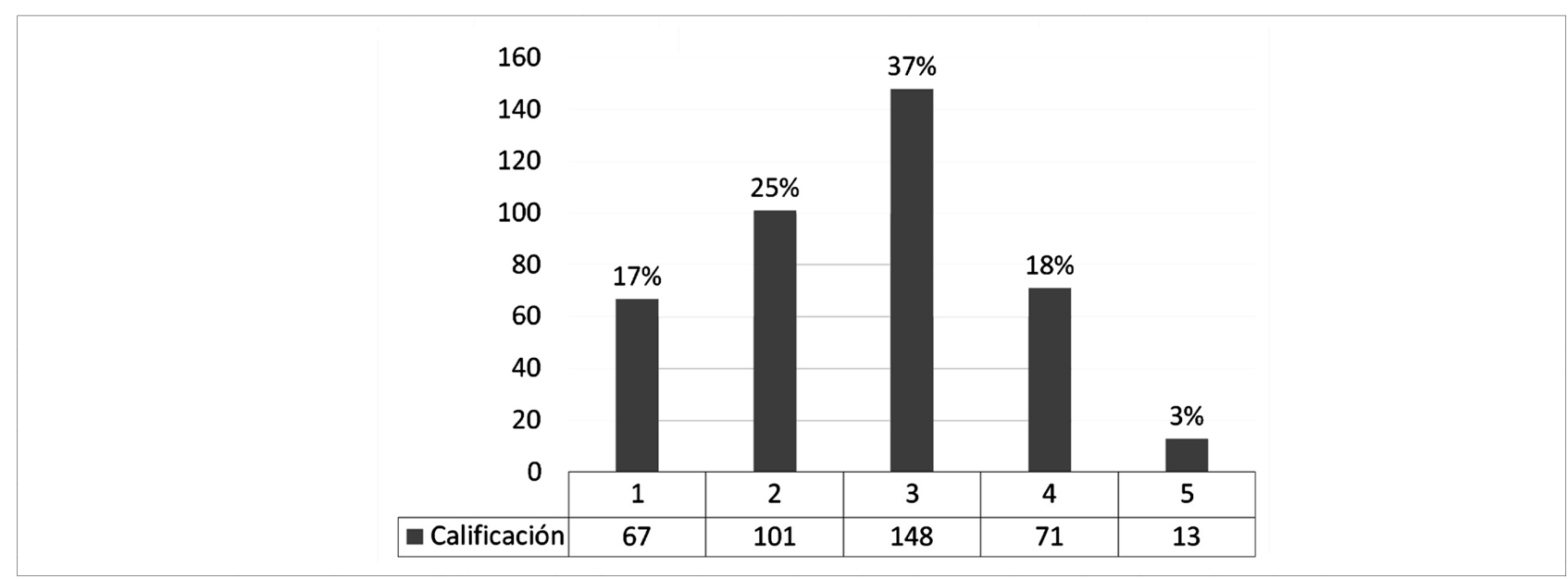

Fuente: Elaboración de los autores.

En cuanto a ¿cómo considera que es el comportamiento de los peatones al transitar por la ciudad? Los resultados definen que el respeto entre peatones se puede resumir diciendo que mientras aproximadamente el 31\% (respuestas 1 y 2 ) califica como deficiente este aspecto, el 29\% (respuestas 4 y 5) lo califica como adecuado. La calificación promedio de todos los encuestados es de 2.94 .

Al solicitar evaluar el respeto que el peatón tiene hacia los conductores, el $42 \%$ (respuestas 1 y 2) de la población piensa que es muy bajo el respeto del peatón a los conductores, mientras el 20\% piensa que sí existe un adecuado respeto. El promedio obtenido global es de 2.69 .

\section{Figura 4. Percepción del respeto que los peatones tienen hacia los conductores}

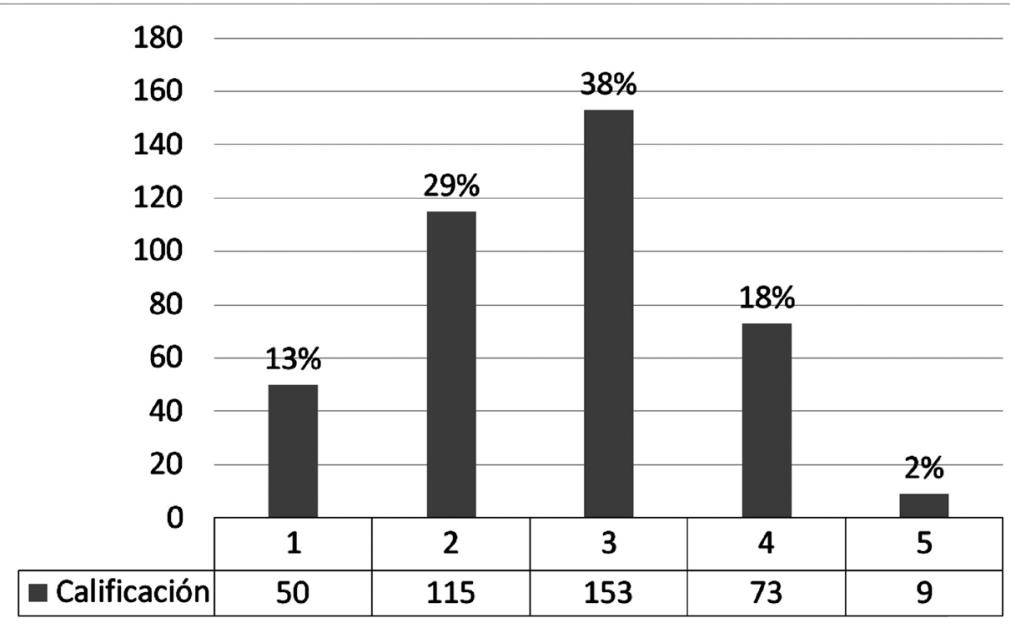

Fuente: Elaboración de los autores. 
El respeto a las señales de tránsito por parte de los peatones, también tiene una muy baja calificación por parte de los encuestados, ya que la percepción del 51\% de encuestados (respuestas 1 y 2), es que hay muy poco o ningún respeto a las señales de tránsito. Se debe tomar en cuenta que los peatones también pueden ser multados por irrespetar señales de tránsito. El promedio global a esta evaluación es de 2.5, el segundo más bajo de los peatones.

En relación a la responsabilidad de la seguridad vial, el 75\% de los encuestados consideran que es de todos, la responsabilidad de la seguridad vial, sin embargo, un 15\% piensa que son las entidades gubernamentales de movilidad, los directos responsables.

Al consultar respecto a la suficiencia de educación, para la prevención de accidentes de tránsito, el 89\% de los encuestados manifestaron que es insuficiente la educación respecto a la prevención de accidentes de tránsito. Finalmente, la pregunta sobre el factor que considera de mayor importancia para lograr un cambio de conductas, actitudes y comportamientos, para mejorar la seguridad vial, la mayoría de encuestados, el 59\%, considera que la educación es el factor de mayor impacto para lograr un cambio de conducta en la ciudadanía, seguido del 20\% que solicita mayor información.

\section{Figura 5. Percepción de la responsabilidad de la seguridad vial}

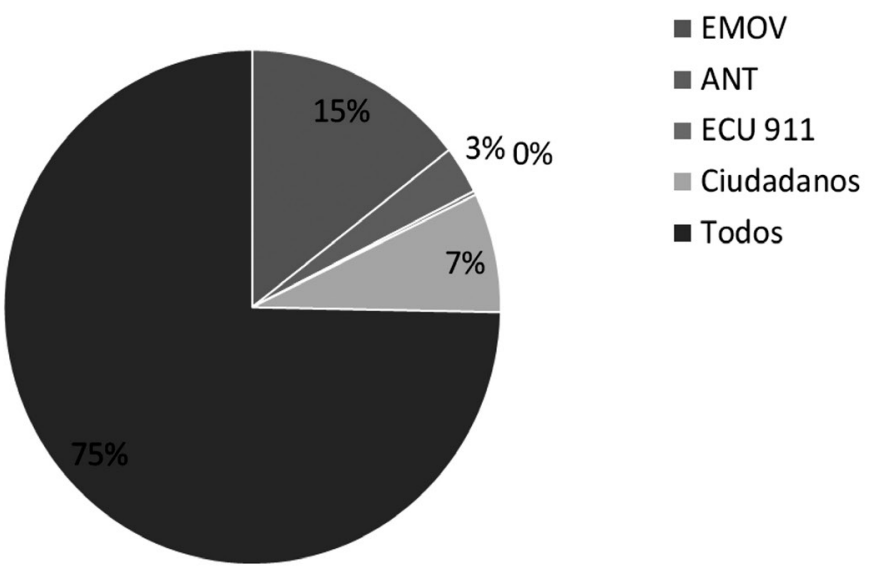

Fuente: Elaboración de los autores.

\subsection{Resultados de la investigación cualitativa}

Mediante la investigación cualitativa se tiene un primer acercamiento a la percepción y el pensamiento de la ciudadanía. Los resultados determinan que los adoptantes objetivo tienen buena predisposición hacia el tema de la seguridad vial, con lo que se facilita la aceptación de los mensajes a comunicar respecto a seguridad vial. Sin embargo, al mencionar campañas sociales enfocadas en la seguridad vial no existe el recordatorio marcado sobre ninguna. En este caso, no existe una campaña social con un fuerte posicionamiento en la percepción del adoptante objetivo.

No existe un agente de cambio con un alto reconocimiento como promotor de seguridad vial en Cuenca. Aunque se tiene presencia en varios medios, no se da la validación de la información emitida por los agentes de cambio por parte del adoptante objetivo. 
Los mensajes emitidos por los agentes de cambio son fáciles de comprender por la ciudadanía. La mayoría de entrevistados recordó por lo menos un mensaje vinculado con la prevención de accidentes de tránsito terrestre, en campañas actuales y anteriores. Pero, la receptividad hacia las campañas sociales de prevención es limitada, pues, por citar un ejemplo, todos comprenden los mensajes de respeto a los límites de velocidad, a no usar el teléfono móvil mientras conduce, entre otros, pero la acción solicitada en los mensajes emitidos por los agentes de cambio se ejecuta de manera poco efectiva por el adoptante objetivo.

$\mathrm{Al}$ momento de realizar las preguntas específicas del cuestionario para las entrevistas a profundidad, los entrevistados dan cuenta de ciertas condiciones básicas de las campañas de prevención vial. Por ejemplo, al preguntar por su recordación respecto a campañas vinculadas con la prevención de accidentes de tránsito, la mayoría de entrevistados recuerdan "alguna campaña", pero con un nulo nivel de recordación de un tema de campaña específico. Algunas respuestas relevantes fueron:

Sí. Una vez vi en el centro, unos jóvenes disfrazados de mimos, que promovían el respeto al paso cebra. (Hombre, 35 años)

Sí. No se estacionen en las paradas de los buses (Mujer, 30 años)

Sí. Pero no me acuerdo bien. (Mujer, 32 años)

Posteriormente, al preguntar por la opinión sobre los mensajes emitidos en las campañas de prevención vial, los entrevistados concluyen que no hay una correcta generación de los mensajes. Algunas respuestas representativas dan cuenta de la situación:

En las campañas les falta llegar a las personas. (Mujer, 35 años)

Los mensajes son interesantes, pero deben hacer más difusión. (Mujer, 30 años)

Sí es motivante, en la radio pasan esos mensajes todo el tiempo con términos que se utilizan aquí. (Mujer, 32 años)

Ante el desconocimiento generalizado de los entrevistados sobre las campañas de prevención vial, se les preguntó por cómo creen ellos que deberían ser emitidos los mensajes por parte de los agentes de cambio. Las respuestas más importantes dan cuenta de los siguientes aspectos:

Más colores, una frase que realmente motive y llene. (Mujer, 30 años)

Con más difusión vial, en vallas y con fotografías gigantes. (Hombre, 27 años)

Usaría personajes como si fuese una telenovela. (Mujer, 26 años)

Deben ir a escuelas, hay personas que no conocen las señales de tránsito y su significado.

(Hombre, 30 años)

En términos generales, las respuestas son coincidentes y giran en torno a mejorar elementos de las comunicaciones, especialmente en los que tienen relación con imágenes, colores, señales de tránsito, entre otros. De esta manera, las respuestas más relevantes de la investigación cualitativa evidencian que:

- Los conceptos en teoría y la información están claros respecto a la importancia de la prevención y la seguridad vial.

- No existe un nivel de recordación alto para el nombre de ninguna campaña de manera específica en la actualidad. Algunos entrevistados aún tenían en mente campañas efectuadas años atrás. 
- En la percepción de la mayoría del adoptante objetivo, no hay información de campañas sociales concernientes a la prevención de accidentes de tránsito.

- La ciudadanía recuerda algunos mensajes y tiene la predisposición de colaborar como ciudadano en los aspectos que consideran importantes.

- En cuanto a la percepción de las campañas, según el criterio de los entrevistados, aún falta mucho más por hacer y las campañas han sido insuficientes, para lograr resultados que perduren a largo plazo.

\section{Conclusiones y discusión}

Luego de la realización de esta investigación, en especial del desarrollo del trabajo de campo, los adoptantes objetivos reconocen que existen campañas sociales sobre prevención de accidentes, sin embargo, los mensajes comunicacionales emitidos por los agentes de cambio, no tienen una buena recepción. El esfuerzo en la comunicación por parte de los agentes de cambio es importante, pero, a pesar de ello, el adoptante objetivo recuerda los mensajes de manera muy dispersa, no se empodera de los mensajes a pesar de comprender la información de los mensajes emitidos, y no se llega a dar la acción propuesta por los agentes de cambio.

La ciudadanía está dispuesta a cumplir las leyes y respetar las normas, sin embargo, se requiere un cambio de actitud por convicción que lleve a modificar el comportamiento que perdure en el tiempo y a medida que avance el proceso de la campaña social, tomar medidas coercitivas de manera gradual.

Son muy pocas las campañas dirigidas hacia el público adulto, con el principal objetivo de prevenir los accidentes de tránsito. Se puede creer que no existieron acciones específicas, emprendidas a nivel de ciudad y probablemente a nivel nacional, o no se logró captar la atención del adoptante objetivo.

Se confirma la poca receptividad por parte del adoptante objetivo hacia las campañas sociales de prevención de accidentes de tránsito en la ciudad de Cuenca.

Aunque hay el interés por la seguridad vial, el nivel de recordación de una campaña específica es muy bajo. Este elemento que tiene relación con el alcance de las campañas, sin embargo, no está directamente vinculado a la receptividad por parte del adoptante objetivo. Pero sí se puede observar en el comportamiento de los individuos y su actuar dentro del grupo social, que aunque existan ciertos cambios de actitudes, aún falta mucho por hacer para lograr un verdadero cambio de conducta que demuestre un mayor desarrollo social.

En la investigación cualitativa se observa que los mensajes que recuerda la ciudadanía son muy dispersos en cuanto a la prevención de accidentes de tránsito. En lo referente a mensajes relacionados a seguridad vial infantil, son escasos y ha llegado muy poco a la percepción de los ciudadanos.

La ciudadanía tiene buena predisposición hacia el tema, con lo que se facilita la aceptación de los mensajes a comunicar respecto a seguridad vial. Se podría también apreciar que existe cierto nivel de conciencia sobre la gravedad de sufrir un accidente de tránsito.

Se hace alusión a varias campañas sociales relacionadas a la seguridad vial, sin embargo, no existe el recordatorio marcado sobre ninguna. Aunque no se puede medir el alcance de las campañas por el nivel de recordación, sí es un parámetro importante. 
De acuerdo a la investigación cualitativa, no existe una campaña social con un fuerte posicionamiento en la percepción del adoptante objetivo.

Los mensajes emitidos por los agentes de cambio son fáciles de comprender para la ciudadanía, la mayoría de entrevistados recordó por lo menos un mensaje relacionado a prevención de accidentes de tránsito terrestre, en campañas actuales y anteriores, pero la receptividad hacia las campañas sociales de prevención de accidentes de tránsito es limitada. Por citar un ejemplo, los adoptantes objetivos comprenden los mensajes de respeto a los límites de velocidad, a no usar el teléfono móvil mientras conduce, a no consumir alcohol si va a conducir. En contraposición, la acción solicitada en los mensajes emitidos por los agentes de cambio, se ejecutan de manera poco efectiva.

Existe la percepción de que no ha existido un buen manejo de medios, de que las imágenes, relatos y gráficas no impactan. Todo elemento debe transmitir, identificarse emocionalmente con los individuos, hablar por sí mismos, generar sentimientos, lograr ser parte de lo que busca el adoptante objetivo y que éste se involucre con la causa de la campaña.

La ciudadanía está dispuesta a cumplir las leyes y respetar las normas, pero se requiere un cambio de actitud por convicción que lleve a modificar el comportamiento que perdure en el tiempo y, a medida que avance el proceso de la campaña social, tomar medidas coercitivas de manera gradual.

La percepción de que no existen controles adecuados, para la prevención de accidentes de tránsito es bastante alta. Este factor aporta al concepto de que la solución no está en manos únicamente de la publicidad social. Los cambios de conducta perdurables son los que se logran por convicción, empero, probablemente por factores culturales, se requiere el apoyo de entidades de control.

Los adoptantes objetivos, a pesar de entender los mensajes emitidos por los agentes de cambio, no generan un cambio de actitud. En este sentido es de trascendental importancia anotar que la ciudadanía no está visibilizando el problema, no dimensiona su magnitud, y no lo reconoce como propio.

Desde luego, no se espera que tan solo con la publicidad social se solucionen todos los problemas existentes tanto en el ámbito del desempeño de las campañas sociales como en los problemas sociales existentes. Sin embargo, a través de estas acciones se puede llegar a ser un dinamizador del cambio social para la adopción de nuevas conductas, y así, en algún momento vivir diferentes realidades en una sociedad que todos quieran formar parte y sentirse orgullosos.

Dar un giro por parte de los agentes de cambio es trascendental, diseñando estrategias con el objetivo de mejorar la percepción de la ciudadanía acerca de las campañas sociales realizadas sobre prevención de accidentes de tránsito en Cuenca, y ejecutando acertadamente estas estrategias.

\section{Referencias}

Alvarado, M. (2005). La Publicidad Social: concepto, objeto y objetivos. Revista de Estudios para el Desarrollo Social de la Comunicación, 2, 265-284. https://bit.ly/3dWEk2L

Alvarado, M. (2009). ¿Publicidad social? Usos y abusos de "lo social" en la publicidad. Revista Icono 14, (13), 125-151. https://bit.ly/2B0idcY

Alvarado, M. (2012). La publicidad en el marco de la comunicación para el desarrollo: hacia un nuevo modelo de publicidad para el cambio social. Cuadernos de Información y Comunicación, 17, 
191-207. https://doi.org/10.5209/rev_CIYC.2012.v17.39264

Arely, C., \& Martín, T. (2017). Representation of the other in social advertising: Analysis of the graphic advertising of NGDOs in social networks. Revista Latina de Comunicación Social, 72, 415-429. https://doi.org/10.4185/RLCS-2017-1172

Arroyo, I. (2014). Publicidad social eficaz desde la universidad: los aspectos experimentales. Zer, 19(37), 191-211. https://bit.ly/2UCbX2b

Aydin, G., \& Koc, E. (2016). Social marketing analysis of attitude toward compulsory earthquake insurance in Turkey. Yönetim ve Ekonomi, 23(2), 389-407. https://bit.ly/2Av0PgC

Baños, M., \& Rodríguez, T. (2009). Desarrollo de un modelo de predicción de la eficacia para la publicidad social. Revista Icono, 14(13), 214-238. https://doi.org/10.7195/ri14.v7i2.324

Barranco, F. (2005). Marketing social corporativo. La acción social de la empresa. ESIC.

Benet, V., \& Nos-Aldás, E. (2003). La publicidad en el tercer sector. Icaria.

Cano, L. (2015). El uso de las figuras literarias en el discurso publicitario. Análisis de los recursos retóricos en la publicidad social. [Trabajo final de máster, Universidad Jaume I, Departamento de Ciencias de la Comunicación].

Dagron, A. (2011). Comunicación para el cambio social: clave del desarrollo participativo. Signo y Pensamiento, 30(58), 26-39. https://bit.ly/2MVjhSd

Dueñas, S., Perdomo, J., \& Villa, L. (2015). El concepto de consumo socialmente responsable y su medición. Una revisión de la literatura. Estudios Gerenciales, 30(132), 287-300. https://doi.org/10.1016/j.estger.2014.01.022

Eguizábal, R. (2009). Industrias de la conciencia, una historia social de la publicidad en España. Península.

French, J., Merritt, R., \& Reynolds Lucy. (2011). Social marketing casebook. Sage Publications.

Gómez, A., \& Quintanilla, I. (1988). Marketing social, desarrollo, concepto y aplicación. Editorial Promolibro.

Gumucio-Dagron, A. (2011). Comunicación para el cambio social: clave del desarrollo participativo. Signo y Pensamiento, 30(58), 26-39. https://bit.ly/30J3Lke

Kotler, P., Hessekiel, D., \& Lee, N. (2013). Lo bueno funciona. LID.

Kotler, P., \& Roberto, E. (1992). Marketing Social, estrategias para cambiar la conducta pública. Díaz de Santos.

Kotler, P., \& Zaltman, G. (1971). Social Marketing: An Approach to Planned Social Change. Journal of Marketing, 35, 3-12. https://doi.org/10.2307/1249783

Leal, A. (2000). Gestión del Marketing Social. McGraw-Hill.

López-Medina, J. (Comp.) (2019). Estrategias de publicidad social. Conyunturas sociales como oportunidad de mejoramiento de valor responsable. Fondo Editorial Universidad Católica Luis Amigó.

Martínez, J. (1998). Publicidad para causas sociales: argumentos racionales vs argumentos emocionales. Comunicación y Sociedad, XI(1), 69-90. https://bit.ly/3fkfRVk

Ministerio del Interior (2014). https://bit.ly/3fkAZe8

Moliner, M. (1998). Marketing social, la gestión de las causas sociales. ESIC.

Muñoz, Y. (2001). El mercadeo social en Colombia. Editorial Universidad EAFIT.

Orozco, J. (2010). Comunicación estratégica para campañas de publicidad social. Pensar la publicidad, 4(2), 169-190. https://bit.ly/2UDFNDn

Orozco-Toro, J., \& Ferré-Pavia, C. (2015). La difusión de las causas sociales y su impacto en la percepción de la imagen de marca. El caso español de Televisión de Catalunya. Cuadernos.info, 1(36), 125-138. https://doi.org/10.7764/cdi.36.551

Orozco-Toro, J., \& Muñoz-Sánchez, O. (2019). Publicidad con sentido social. Editorial UPB.

Pérez, L. (2006). Marketing social, teoría y práctica. Prentice Hall.

Pinilla, M. (2011). Contribución del marketing social a la sostenibilidad de fundaciones sin ánimo de lucro: un estudio de caso. [Tesis de maestría, Universidad Nacional de Colombia]. https://bit.ly/3hjn1el

Rodrigo, I., \& Rodrigo, L. (2013). La causa social en la creatividad publicitaria: Valores y confianza. Los discursos publicitarios en situaciones de crisis. Pensar la Publicidad, 7(2), 253-274. https://doi.org/10.5209/rev_PEPU.2013.v7.n2.46177

Rosario-Ubiera, R. (2014). Publicidad solidaria: eficacia de la publicidad televisiva de manos unidas. Prisma Social, 13, 610-651. https://bit.ly/37o1mgc 
Saghi, Z., \& Lotfabadi, V. (2016). The Effect of Social Marketing on Customer Satisfaction with Regard to the Moderating Role of Environmental Approach. International Journal of Management, Accounting and Economics, 3(6), 378-389.

Saiz, V., Alvarado, M., \& Gómez, P. (2018). Advocacy of trafficking campaigns: A controversy story. Comunicar, 55, 29-38. https://doi.org/10.3916/C55-2018-03

Sar, S., \& Anghelce, G. (2015). Congruity between mood and message regulatory focus enhances the effectiveness of antidrinking and driving advertisements: a global versus local processing explanation. International Journal of Advertising, 34(3), 421-446. https://doi.org/10.1080/02650487.2014.996198

Taricco, J. (2009). De la publicidad de "bien público" a la publicidad social en Argentina. Revista Icono, 14(13), 24-36. https://doi.org/10.7195/ri14.v7i2.315

Vergara, E., \& Rodríguez, M. (2010). El impacto social y cultural de la publicidad entre los jóvenes chilenos. Comunicar Revista Científica de Educomunicación, 18(35), 113-119.

https://doi.org/10.3916/C35-2010-03-04 